\title{
ON THE EXPLOSION OF CHAIN-THERMAL REACTIONS
}

\author{
R. O. AYENI'
}

(Received 6 June 1980; revised 26 October 1981)

\begin{abstract}
A chain reaction of oxygen (reactant) and hydrogen (active intermediary) with nitrosyl chloride (sensitizer) as a catalyst may be modelled mathematically as a non-isothermal reaction. In this paper we present an asymptotic analysis of a spatially homogeneous model of a non-isothermal branched-chain reaction. Of particular interest is the so-called explosion time and we provide an upper bound for it as a function of the activation energy which can vary over all positive values. We also establish a bound on the temperature when the activation energy is finite.
\end{abstract}

\section{Introduction}

We consider a model of a non-isothermal branched chain reaction. In particular we consider a vessel which is filled with a potentially reactive mixture containing a reactant $A$, an active intermediary $C$ and a sensitizer $M$. We assume that the reaction satisfies

$$
\begin{aligned}
A+C & \rightarrow(1+n) C+\text { products, } \\
C+M+M & \rightarrow \text { products or stable molecules, }
\end{aligned}
$$

where $n$ is a natural number. The reaction (1.1) is a propagation step, while reaction (1.2) is a termination step.

Recently Kapila [5] investigated a model of a non-isothermal branched chain reaction. Instead of our reaction (1.2) Kapila assumed that the termination step is

$$
C \stackrel{\text { wall }}{\rightarrow} \text { stable species. }
$$

\footnotetext{
'Department of Mathematics, University of Ife, Ile-Ife, Nigeria. (c) Copyright Australian Mathematical Society 1982
} 
Also recently Ayeni [1] investigated sensitized chain-thermal reactions assuming that the termination steps are

$$
\begin{aligned}
C & \rightarrow \text { wall, } \\
C+C+M & \rightarrow M+\text { products. }
\end{aligned}
$$

One parameter of importance in this type of reaction is $R T_{0} / E$ (where $R$ is the universal gas constant, $T_{0}$ is the initial temperature of the system and $E$ is the activation energy). If $R T_{0} / E$ is small, then an investigator may seek to solve the system of equations asymptotically in the limit $R T_{0} / E \rightarrow 0$. Kapila and Ayeni took this approach. They assumed that $0 \leqslant R T_{0} / E \leqslant 0.05$.

However, a look at a table of activation energies of the propagation and termination stage (see Dainton [3], page 53) shows that there are situations when the activation energy is as high as $18 \mathrm{kcal}$. and as low as $1.2 \mathrm{kcal}$. In the same table one sees that the activation energy of the termination step ranges between 0 and $5.2 \mathrm{kcal}$. If we assume an initial temperature of $300^{\circ} \mathrm{K}$, an activation energy of $18 \mathrm{kcal}$. gives a number $\sim 0.033$ for $R T_{0} / E$, while $R T_{0} / E \sim 0.50$ when $E$ is 1.2 kcal. While the analyses of Kapila and Ayeni mentioned above are quite valid for reactions of the first type, a new investigation needs to be carried out for the reactions which are close to the second type.

Thus in this analysis we do not assume as the basis that $R T_{0} / E \rightarrow 0$, but we assume:

(i) the surface is inactive catalytically,

(ii) a small concentration of the chain carriers is already present in the starting mixture,

(iii) the propagation rate varies linearly as $Y_{C} Y_{A} \exp (-E / R T) / T^{2}$ (see Dainton [3], page 57), where $Y_{C}$ is the mass fraction of the active centre, $Y_{A}$ is the mass fraction of the reactant, $T$ is the temperature of the mixture, $E$ is the activation energy and $R$ is the universal gas constant,

(iv) the termination step (1.2) does not depend on temperature. In practice, the step (1.2) is weakly dependent on temperature, that is, the termination step varies inversely as $T^{l}$ where $l$ is a positive number (see, for example, Kondratiev [6]). Since in this paper we do not assume that $E$ is necessarily large, assuming that the reaction (1.2) does not depend on temperature means that $0<l \ll 1$,

(v) the system is spatially homogeneous. This appears to be reasonable, since we are assuming an inactive surface and

(vi) the overall reaction is exothermic, with $d T / d t$ directly proportional to $Y_{C} Y_{M}^{2}$. Notice that in the hydrogen-oxygen system the constant of proportionality is approximately unity ([5]).

The mathematical equations governing the reactive system (1.1) and (1.2) are those of species and heat conservation (see, for example, Kapila [5], Kondratiev 
[6], Dainton [3] and Ayeni [1]), and they are defined (bearing the above assumptions in mind) by:

$$
\begin{gathered}
d Y_{A} / d \tau=-B_{1} \rho Y_{A} Y_{C} \nu^{2} h^{2} \exp (-E / R T) /\left(W_{C} k^{2} T^{2}\right), \\
d Y_{C} / d \tau=n B_{1} \rho Y_{A} Y_{C} \nu^{2} h^{2} \exp (-E / R T) /\left(W_{A} k^{2} T^{2}\right)-B_{2} Y_{C} Y_{M}^{2}, \\
\left(C_{P} / Q\right) d T / d \tau=q B_{2} Y_{C} Y_{M}^{2} \\
T(0)=T_{0}, \quad Y_{A}(0)=Y_{A_{0}}, \quad Y_{C}(0)=Y_{C_{0}},
\end{gathered}
$$

where the nomenclature is

$$
\begin{aligned}
& Y . \text { mass fraction } \\
& W \text { molecular weight } \\
& \rho \text { density } \\
& Q \quad \text { heat release per unit mass } \\
& R \text { universal gas constant } \\
& B \text { pre-exponential factor } \\
& q \text { positive number } \\
& n \text { natural number } \\
& C_{P} \text { specific heat at constant pressure } \\
& \tau \text { dimensional time } \\
& T \text { temperature } \\
& \nu \text { vibration frequency } \\
& h \text { Planck's constant } \\
& k \text { Boltzmann's constant. }
\end{aligned}
$$

The subscripts used have the following meanings:

$$
\begin{aligned}
& O \text { initial } \\
& A \text { reactant } \\
& M \text { active centre } \\
& 1 \text { in propagation step (1.1) } \\
& 2 \text { in termination step (1.2). }
\end{aligned}
$$

Notice that the units of the symbols are as in [2], [3] and [4].

\section{Method of solution}

Let

$$
\begin{aligned}
a & =B_{2} Y_{M}^{2} / t_{1} \\
\varepsilon \lambda & =Y_{C_{0}} Q /\left(T_{0} C_{P}\right) \\
\alpha & =R T_{0} / E
\end{aligned}
$$




$$
\begin{aligned}
t_{1} & =n B_{1} \rho Y_{A_{0}} \exp \left(-E / R T_{0}\right) / W_{A} \\
t & =\tau t_{1}=\operatorname{dimensionless} \text { time } \\
\theta & =\left(T-T_{0}\right)\left(E / R T_{0}^{2}\right)=\text { dimensionless temperature } \\
\delta & =Y_{A_{0}} n W_{C} Q K^{2} T_{0} /\left(W_{A} C_{P} \nu^{2} h^{2}\right) \\
\varepsilon & =\exp (-1 / \alpha) \\
b / \varepsilon & =q B_{2} Y_{M}^{2} /\left(t_{1} \alpha\right) \\
y & =Y_{A} n W_{C} Q k^{2} T_{0} /\left(W_{A} C_{P} \nu^{2} h^{2}\right)=\text { scaled reactant } \\
z & =Y_{C} Q /\left(T_{0} C_{P}\right)=\text { scaled active centre. }
\end{aligned}
$$

Notice that we have neglected the diffusion terms in equations (1.3)-(1.5) following the suggestion of Dainton ([3], page 103). Then the dimensionless versions of (1.3)-(1.6) are:

$$
\begin{aligned}
& d y / d t=-(z y / \delta)\left(1 /(1+\alpha \theta)^{2} \exp (\theta /(1+\alpha \theta))\right), \\
& d z / d t=(z y / \delta)(1 /(1+\alpha \theta))^{2} \exp (\theta /(1+\alpha \theta))-a z, \\
& d \theta / d t=b z / \varepsilon, \\
& \theta(0)=0, \quad z(0)=\varepsilon \lambda, \quad y(0)=\delta .
\end{aligned}
$$

It is worth noting that the exact integral is $(a \varepsilon / b) \theta+y+z=\delta+\varepsilon \lambda$.

We now seek to solve (2.1)-(2.4) asymptotically in the limit $\varepsilon \rightarrow 0$. The appropriate expansions for $\theta, z$ and $y$ which are suggested by the equations (2.1)-(2.3) and the initial conditions (2.4) are:

$$
\begin{aligned}
& \theta=\theta_{0}+\varepsilon \theta_{1}+\text { h.o.t., } \\
& z=\varepsilon z_{0}+\text { h.o.t., } \\
& y=\delta+\varepsilon y_{1}+\text { h.o.t. }
\end{aligned}
$$

where h.o.t. reads "higher order terms in $\varepsilon$ ". In our analysis we are interested only in the first terms. This means that we want to investigate the behaviour of $\theta_{0}$ and $z_{0}$. Putting the expansions (2.5)-(2.7) into the equations (2.4) we find that:

$$
\begin{aligned}
& d z_{0} / d t=z_{0} \exp \left(\theta_{0} /\left(1+\alpha \theta_{0}\right)\right)-a z_{0}, \\
& d \theta_{0} / d t=b z_{0}, \\
& \theta_{0}(0)=0, \quad z_{0}(0)=\lambda, \quad d \theta_{0} / d t=b \lambda \text { at } t=0 .
\end{aligned}
$$

By differentiating equation (2.9) with respect to $t$, substituting for $d z_{0} / d t$ and then integrating the resulting expression, one finds that

$$
\begin{aligned}
& d \theta_{0} / d t=\exp \left(\theta_{0} /\left(1+\alpha \theta_{0}\right)\right)-a \theta_{0}-1+\lambda b, \\
& \theta_{0}(0)=0 .
\end{aligned}
$$


One notes further that (2.11) implies

$$
\begin{aligned}
& z_{0}=\left(\exp \left(\theta_{0} /\left(1+\alpha \theta_{0}\right)\right)-a \theta_{0}-1+b \lambda\right) / b, \\
& z_{0}(0)=\lambda .
\end{aligned}
$$

The basic questions in this analysis are:

(1) what is the effect of $\alpha\left(=R T_{0} / E\right)$ on $\theta_{0}$ and $z_{0}$ ?

(2) how does $\theta_{0}$ affect $z_{0}$ ? Physically this means that we want to compare isothermal reaction and non-isothermal reaction, and

(3) what is the effect of the sensitizer and the temperature and the active centre?

We will answer the above questions in the next section.

\section{Behaviour of solution}

In Figures 1 and 2 we display the graphs of $z_{0}$ versus $\theta_{0}$ for various values of $\alpha$, $a, b$ and $\lambda$. It is easy to check that $z_{0}$ is a convex function of $\theta_{0}$ if $\alpha \geqslant 0.5$ and that it is a concave function of $\theta_{0}$ if $\alpha \rightarrow 0$ provided $b>0$. If $\alpha \geqslant 0.5, z_{0}$ vanishes at a finite value $\theta_{0_{\infty}}$ of $\theta_{0}$. Equation (2.11) then shows that $\theta_{0} \rightarrow \theta_{0_{\infty}}$ as $t \rightarrow \infty$. It is worth pointing out that $\theta_{0}$ can tend to a finite value as $t$ tends to infinity as shown in Figure 2(iii) even when $\alpha \rightarrow 0$, but in this case the value depends not only on $b$ being greater than zero but also on $a$ and $\lambda$. Also we note from Figure 1(i) that $z_{0}$ can still be a convex function of $\theta_{0}$ if $0<\alpha<0.5$ but this behaviour depends on $\lambda$ and $a$ as well.

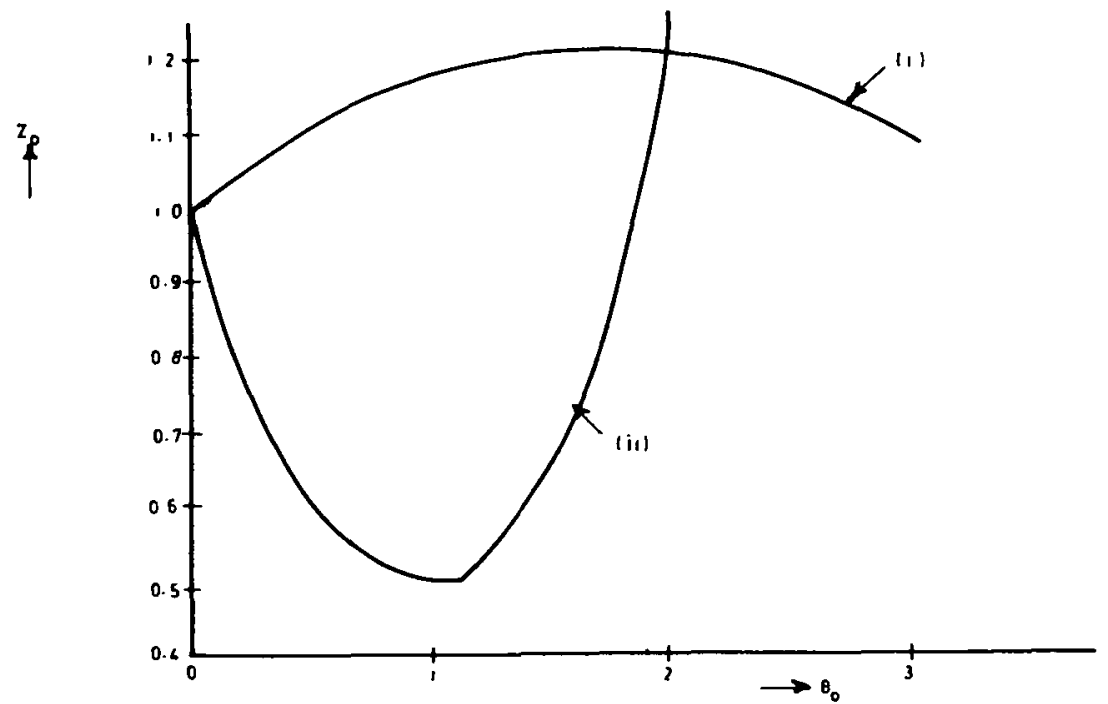

Figure 1. Plots of $z_{0}$ against $\theta_{0}$ when $\alpha \neq 0$. (i) $b=1, \lambda=1, a=0.81, \alpha=0.46$, (ii) $b=1, \lambda=1$, $a=2, \alpha=0.1$. 


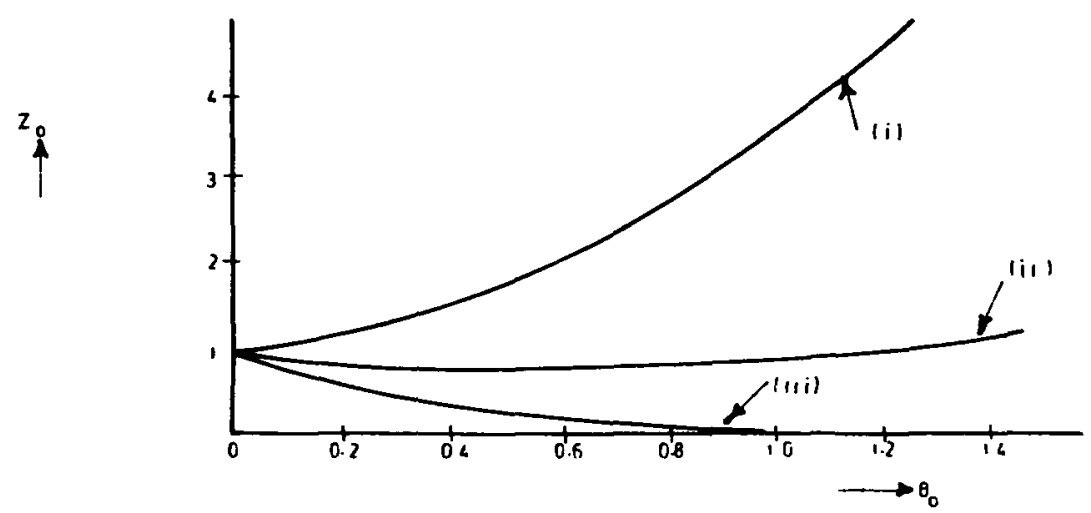

Figure 2. Plots of $z_{0}$ against $\theta_{0}$ when $\alpha=0$. (i) $b=0.5, a=0.5, \lambda=1$, (ii) $b=2, a=2, \lambda=1$, (iii) $b=1, a=e, \lambda=1$.

Let us now examine the effects of $\alpha$ on $z_{0}$ and $\theta_{0}$ individually. First we note from equation (2.8) that if $\theta_{0}=0$ (isothermal reaction), then

$$
z_{0}=\lambda \exp ((1-a) t)
$$

so that the behaviour of $z_{0}$ depends mainly on $a$. When $\theta_{0}$ is not a constant we may use upper and lower solutions to bound the growth of $z_{0}$ and $\theta_{0}$. Consider the differential equation

$$
\begin{gathered}
d u / d t=f(u) \quad(t>0), \\
u(0)=u_{0} .
\end{gathered}
$$

By lower and upper solutions we mean a pair of smooth functions $v(t)$ and $w(t)$ such that for every finite $T>0, w(t) \geqslant v(t)$ on $(0, T]$ and $v(t)$ satisfies the inequalities

$$
d v / d t \leqslant f(v), \quad v(0) \leqslant u_{0},
$$

while $w(t)$ satisfies the reverse inequalities in (3.1). If $v(t)$ and $w(t)$ exist, then $v(t) \leqslant u(t) \leqslant w(t)$ (see [9]).

We note that if $\alpha \rightarrow 0$, the maximum value of $\exp \left(\theta_{0} /\left(1+\alpha \theta_{0}\right)\right)$ is $\exp (1 / \alpha)$. Thus in this case, $\lambda$ and $\lambda \exp (\exp (1 / \alpha) t)$ are lower and upper solutions for $z_{0}$. That is,

$$
\lambda \leqslant z_{0} \leqslant \lambda \exp ((\exp 1 / \alpha-a) t) .
$$

In this same situation $(\alpha \nrightarrow 0)$

$$
0 \leqslant \theta_{0}(t) \leqslant((\exp (1 / \alpha)+\lambda b-1) / a)(1-\exp (-a t)) .
$$


It is significant to note that when upper and lower solutions are known we can use them to estimate upper and lower bounds for the explosion time. It is this approach that we intend to use in what follows to estimate the upper bound for the explosion time when $\alpha \rightarrow 0$. When $\alpha \rightarrow 0$, equation (2.8) becomes

$$
\left.\begin{array}{l}
d z_{0} / d t=z_{0} \exp \left(b \int_{0}^{t} z_{0}(x) d x\right)-a z_{0}, \\
z_{0}(0)=\lambda .
\end{array}\right\}
$$

A lower solution (which becomes a lower bound once an upper solution is found) when $0 \leqslant a \leqslant 1$ is

$$
u(t)=\lambda \sec ^{2}\left((\lambda b / 2)^{1 / 2} t\right) .
$$

It is trivial to prove that $u(t) \rightarrow \infty$ as $t \rightarrow \pi(2 \lambda b)^{-1 / 2}$. We claim that $u(t)$ is indeed a lower bound for $z_{0}$, but we see no point in finding an upper solution in this analysis (which actually exists) since the method of approach is clearly written in [9]. What we have now is that

$$
z_{0}(t) \geqslant \lambda \sec ^{2}\left((\lambda b / 2)^{1 / 2} t\right)
$$

and (3.4) implies that

$$
\theta_{0}(t) \geqslant(2 \lambda b)^{1 / 2} \tan \left((\lambda b / 2)^{1 / 2} t\right) .
$$

Explosion therefore occurs prior to time $\pi(2 \lambda b)^{-1 / 2}$.

Let us compare this bound with the so-called quadratic time of explosion. If we assume the quadratic expression for $\exp (\theta)$, that is,

$$
\exp (\theta)=1+(e-2) \theta+\theta^{2}
$$

then

$$
\begin{gathered}
\theta_{0}(t)=h \frac{\{(e-2-a) /(2 h)+\tan (h t)\}}{1-((e-2-a) /(2 h)) \tan (h t)}-\frac{e-2-a}{2}, \\
z_{0}(t)=h \frac{\left\{h+(e-2-a)^{2} /(4 h)\right\} \sec ^{2}(h t)}{b\{1-((e-2-a) /(2 h)) \tan (h t)\}^{2}},
\end{gathered}
$$

where $h^{2}=\lambda b-((e-a-2) / 2)^{2}$. If we take $\lambda=1, a=0.22, b=4$, then $\pi(2 \lambda b)^{-1 / 2} \sim 1.11$. The quadratic time of explosion is 0.73 . This confirms that $\lambda \sec ^{2}\left((\lambda b / 2)^{1 / 2} t\right)$ is a lower solution. It is worth pointing out that the quadratic expression for $\exp (\theta)$ is an approximation. Its merits and demerits have been discussed by Gray et al. in [4]. A typical time history of $\theta_{0}\left(z_{0}\right)$ is shown in Figure 3. The singularity in the solution characterizes explosion and the time 0.73 which signals the onset of explosion is known as the induction period (see, for example, [5]). 


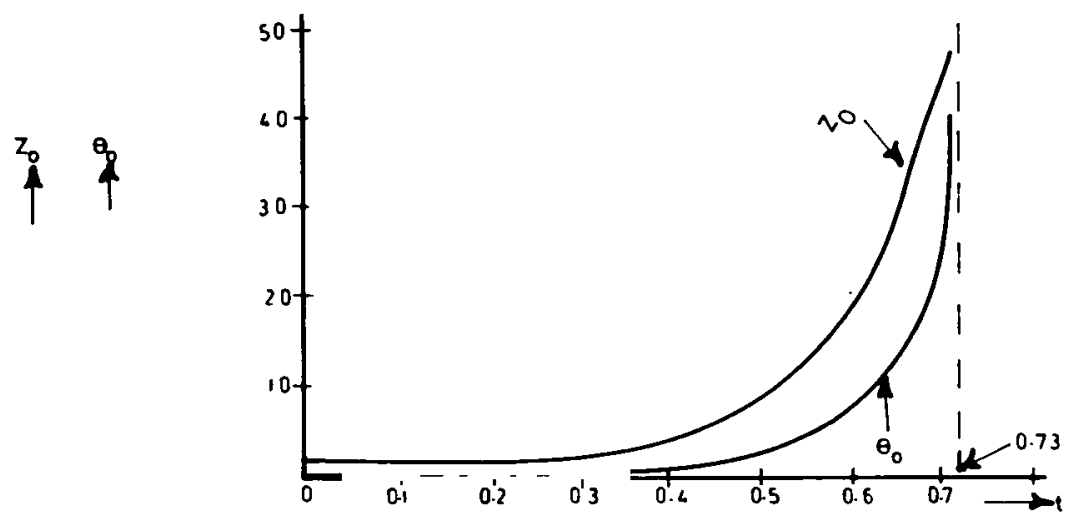

Figure 3. Plots of $z_{0}$ and $\theta_{0}$ against $t$ for $b=4, a=0.22, \lambda=1, \alpha=0$.

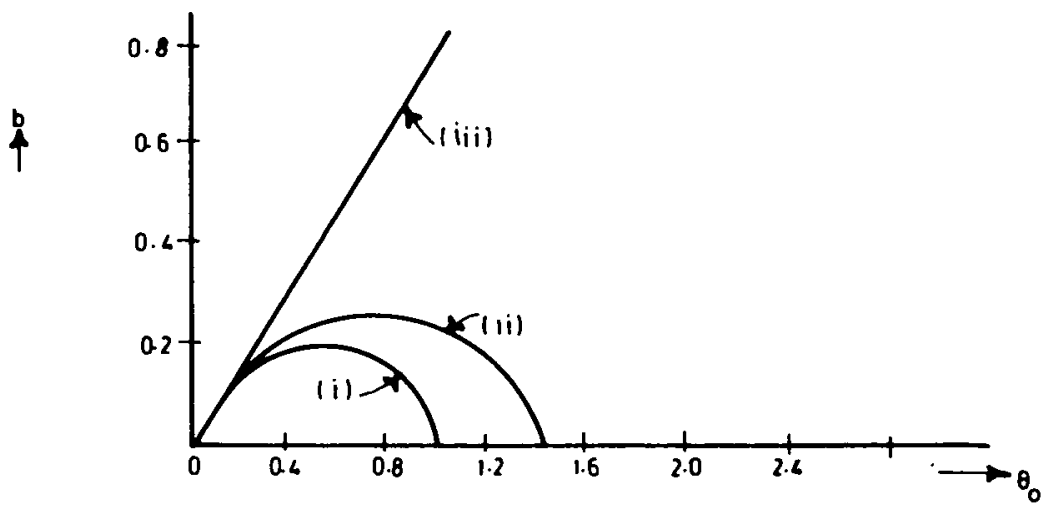

Figure 4. Plots of $b$ against $\theta_{0}$ when $d \theta_{0} / d t=0, \lambda=1, a=e-1$. (i) $\alpha=0$, (ii) $\alpha=0.1$, (iii) $\alpha=0.46$.

To conclude this analysis we examine the relationship between the scaled sensitizer $b$ and the steady state temperature $\theta_{0}$. Figure 4 shows the graph of $b$ as a function of steady temperature $\theta_{0}$. First we notice that if $b$ is zero, then zero is a possible steady temperature. For $\alpha \geqslant 0.5$, it is easy to check that $b$ is a concave function of $\theta_{0}$. Physically this means that there exists a steady temperature if $b$ is large enough, whereas for $0 \leqslant \alpha<0.5$ the existence of steady temperature depends on $\lambda$ and $a$. 


\section{Conclusion}

We have found that chain explosion could still occur even when the reaction is isothermal. In fact the active centre $z_{0}$ is $\exp (3 t / 4)$ if $\lambda=1$ and $a=\frac{1}{4}$. That is, $z_{0} \rightarrow \infty$ as $t \rightarrow \infty$. When the reaction is chain thermal (non-isothermal) chain explosion occurs prior to $t=1.11$ if $\lambda=1, a=0.22, b=4$ and $\alpha \rightarrow 0$. Whereas if the activation energy is finite $(\alpha \rightarrow 0)$ the temperature $\theta_{0}$ satisfies the inequality

$$
0 \leqslant \theta_{0}(t) \leqslant((\exp (1 / \alpha)+\lambda b-1) / a)(1-\exp (a t)) .
$$

Thus thermal explosion cannot occur if $a \nrightarrow 0$.

We have also found that the active $z_{0}$ may increase or decrease as the temperature $\theta_{0}$ increases, its behaviour depends on the parameter $2 \alpha \exp (1 / \alpha) a^{-1 / 2}$.

\section{Acknowledgements}

We thank our referees for their useful suggestions. We are also indebted to Professor I. H. Sloan whose advice has been helpful in putting the paper in a publishable condition.

\section{References}

[1] R. O. Ayeni, "Criteria for branched-chain explosion", Nigerian J. of Natural Sciences (to appear).

[2] T. Boddington, P. Gray and G. C. Wake, "Criteria for thermal explosions with and without reactant consumption", Proc. Roy. Soc. London Ser. A 357 (1977), 403-422.

[3] F. S. Dainton, Chain reactions: an introduction (Wiley, New York, 1966).

[4] P. Gray and M. J. Harper, "Thermal explosions: part 1-induction periods and temperature changes before spontaneous ignition", Trans. Faraday Soc. 55 (1959), 581-590.

[5] A. K. Kapila, "Homogeneous branched-chain explosion: initiation to completion", J. Engrg. Math. 12 (1978), 221-235.

[6] V. N. Kondratiev, The theory of kinetics (Elsevier, New York, 1969), pages 81-188.

[7] G. S. S. Ludford, "Combustion: basic equations and peculiar asymptotics", J. Mécanique 16 (1977), 531-551.

[8] M. F. R. Mulcahy, Gas kinetics (Nelson, London, 1973).

[9] C. V. Pao, "Non existence of global solutions and bifurcation analysis for a boundary value problem of parabolic type", Proc. Amer. Math. Soc. 65 (1977), 245-251.

[10] D. H. Satinger, "A nonlinear parabolic system in the theory of combustion", Quart. Appl. Math. 33 (1975), 47-61. 